\title{
Modelo de gestión de seguridad total en una institución de enseñanza técnica superior para reducir la accidentabilidad
}

\author{
Total safety management model in a higher technical teaching institute to \\ reduce accidentability
}

Mickey Elvis Ayrampo Espinoza ${ }^{1}$

Recibido: 11/08/2020 - Aprobado: 20/04/21 - Publicado: 18/06/2021

\begin{abstract}
RESUMEN
Objetivo: Fue realizar la propuesta de un modelo de gestión de seguridad y salud ocupacional integral en una institución de enseñanza técnica superior que incremente la cultura segura y reduzca la accidentabilidad de sus trabajadores y partes interesadas. Métodos: Estudio aplicado, enfoque cualitativo, no experimental. La muestra estuvo conformada por documentos de investigación y de gestión, de verificación de diagnóstico de las condiciones de riesgos en de la institución de enseñanza técnica superior y encuesta de conocimiento. La técnica de recolección fue documental. Resultados: La evaluación de la gestión en seguridad y salud en el trabajo basado en la norma ISO 45001:2018 presentó un nivel medio de implementación. Respecto al índice de accidentabilidad, el índice de frecuencia y el índice de gravedad presentaron en general un comportamiento decreciente. Asimismo, después de la reevaluación se incrementó en un $88 \%$ de implementación. Conclusiones: La propuesta de gestión de seguridad total está focalizada en dimensiones (física, emocional y social), agentes (alumnos, directivos, familia, administrativo, coordinadores, entidades del entorno) y ámbitos de gestión (la provisión de recursos o presupuesto, la optimización, ciclos o periodos, clima 0 atmósfera positiva y segura en las sedes de estudio, áreas, talleres, las formas de comunicación y relación abierta, segura y de confianza, la gestión de personas, los ambientes e infraestructura de trabajo y estudios y la prevención y protección de accidentes, incidentes y riesgos). Dividida en cuatro componentes: seguridad proactiva, operativa, pasiva y reactiva; poniendo énfasis en una cultura de seguridad total mediante el pensamiento crítico, proactivo y preventivo, analógicamente similar a la calidad total.
\end{abstract}

Palabras clave: modelo de seguridad total ocupacional; institución de enseñanza técnica superior; accidentabilidad.

\begin{abstract}
Objective: It was to carry out the proposal of a comprehensive occupational health and safety management model in a higher technical education institution that increases the safe culture and reduces the accident rate of its workers and stakeholders. Methods: Applied study, qualitative approach, not experimental. The sample consisted of research and management documents, verification of the diagnosis of risk conditions in the institution of higher technical education and a knowledge survey. The collection technique was documentary. Results: The evaluation of occupational health and safety management based on the ISO 45001: 2018 standard presented a medium level of implementation. Regarding the accident rate, the frequency rate and the severity rate generally showed a decreasing behavior. Likewise, after the reevaluation, implementation increased by $88 \%$. Conclusions: The total security management proposal is focused on dimensions (physical, emotional and social), agents (students, managers, family, administrative, coordinators, entities of the environment) and management areas (the provision of resources or budget, the optimization, cycles or periods, positive and safe climate or atmosphere in study venues, areas, workshops, forms of communication and open, safe and trustworthy relationships, people management, work and study environments and infrastructure and the prevention and protection of accidents, incidents and risks). Divided into four components: proactive, operational, passive and reactive security; emphasizing a total safety culture through critical, proactive and preventive thinking, analogous to total quality.
\end{abstract}

Keywords: total occupational safety model; institution of higher technical education; accident rate.

\footnotetext{
1 Egresado de la Maestría en Gestión Integrada en Seguridad, Salud Ocupacional y Medio Ambiente de la Unidad de Postgrado. Facultad de Ingeniería Geológica, Minera, Metalúrgica y Geográfica, Universidad Nacional Mayor de San Marcos, Lima, Perú. Ingeniero Industrial.

E-mail: gerencia@igesperu.com; mayrampo@hotmail.com
} 


\section{INTRODUCCIÓN}

Últimamente, el impulso de los sistemas de seguridad y salud ha ido adquiriendo un protagonismo a nivel mundial, preocupación que no es una casualidad por las altas cifras de personas que mueren debido a los accidentes laborales o de trabajo, enfermedades asociadas a las actividades. Según cifras del OIT, cada día mueren 6300 personas, más de 2,3 millones de muertes por año y 317 millones de accidentes de trabajo, generando un enorme costo para las organizaciones, representando un 4\% del PBI global de cada año (Organización Internacional del Trabajo, 2018). El sector educativo no es ajeno a estas eventualidades, sin contar con cifras exactas sobre los accidentes. En nuestro país no hay una norma de seguridad y salud sectorizada para el ámbito educativo, la mayoría está basada en entornos industriales. La realidad muestra que los accidentes en el trabajo y las enfermedades ocupaciones representan un considerable costo de vidas humanas y un costo económico muy importante con un limitado conocimiento de la magnitud del problema (MTPE \& MINSA, 2010). Por su parte, el SENATI (Servicio Nacional de Adiestramiento para el Trabajo Industrial) en el periodo de 2016 a 2019, se ha experimentado un incremento de accidentabilidad en más del $90 \%$ correspondiente a alumnos, trabajadores y personal contratista. Si bien "INSTITUTO DE FORMACIÓN TÉCNICA SUPERIOR" - (INFOTESU) cuenta con una certificación en seguridad y salud en el trabajo OHSAS 18001 y su pronta migración a la norma ISO 45001:2018 -como parte de un Sistema Integrado de Gestión-, las "revisiones" o "auditorias" que garantizan el desempeño de la SST por sí mismas, pueden no ser suficientes para proporcionar a una organización la seguridad de que su desempeño cumple con la provisión de un entorno laboral seguro y saludable, más aun cuando un solo jefe de seguridad y salud laboral del "INSTITUTO DE FORMACIÓN TÉCNICA SUPERIOR” - (INFOTESU), resulta insuficiente para planificar, programar, ejecutar, supervisar y mejorar la gestión de seguridad y salud en todas las sedes que "INSTITUTO DE FORMACIÓN TÉCNICA SUPERIOR" - (INFOTESU) posee a nivel nacional. Este panorama nos lleva a plantearnos diversos interrogantes, que daremos respuesta en esta investigación, como ¿Qué tan prioritario es la seguridad en una Institución Educativa Técnica Superior? ¿Qué acciones en pro de la prevención de accidentes y enfermedades ocupacionales actualmente desarrolla una Institución Educativa Técnica Superior? ¿Qué aspectos relativos a la seguridad y salud en el trabajo en las Instituciones Educativas Técnicas Superior tienen una normativa aplicable? ¿Qué modelo de sistema de gestión de seguridad total o integral se puede plantear para una Institución Educativa Técnica Superior? El objetivo de este estudio será plantear un modelo de Seguridad Total que implemente una estrategia de seguridad y transformación de una cultura educacional segura, en la institución de enseñanza técnica superior para reducir la accidentabilidad en Lima, Perú en el 2021 (SENATI-Servicio Nacional de Adiestramiento para el Trabajo Industrial, 2012).

\section{MÉTODOS}

El estudio fue aplicado con enfoque cualitativo, no experimental (Hernández-Sampieri, R., FernándezCollado, C. y Baptista-Lucio, 2014). La muestra estuvo conformada por información documentada de origen externo (tesis, libros, artículos científicos) sobre sistemas de gestión de seguridad y salud ocupacional en organizaciones públicas como privadas en el contexto nacional como internacional y documentos internos de gestión de la institución de enseñanza técnica superior. La técnica de recolección fue documental. El análisis documentario, obtuvo dos partes: La primera parte radicó en descubrir una exploración de la teoría y los primordiales modelos teóricos y aplicados sobre gestión de seguridad y salud ocupacional en la literatura nacional como internacional, a fin de que, se puedan identificar las ideas trascendentales y significativas, que nos accedieron catalogar la información y posteriormente describirlos. La segunda parte, consistió en revisar documentos de evaluación sobre la gestión de seguridad y salud ocupacional basada en la norma ISO 45001 en su versión 2018 y proporcionar una perspectiva sobre el contexto actual de la institución de enseñanza técnica superior, además de información estadística relacionada a los accidentes en la institución educativa

\section{RESULTADOS}

\subsection{Diagnóstico de la gestión de seguridad y salud en el trabajo de una institución de enseñanza técnica superior}

El diagnóstico de la evaluación de la gestión de seguridad y salud en el trabajo basado en la norma ISO 45001, para una institución de enseñanza técnica superior, dio como resultado los siguientes hallazgos. Respecto al capítulo de CONTEXTO DE LA ORGANIZACIÓN, se obtuvo una implementación del 51.5\%.

En la comprensión de la organización y su contexto de INFOTESU, no se pudo evidenciar en los asuntos externos la identificación de criterios como el entorno social, cultural, tecnológico, financiero, pandémico y ambiental. Incluyendo la competencia del mercado en toda su geografía. Asimismo, el ingreso de contratistas, sub contratistas, proveedores, entre otros terceros que prestan servicios. Por otro lado, en los asuntos internos, la falta de detección y evaluación de las relaciones con los colaboradores, sus percepciones, valores; los horarios y condiciones de trabajo. Por otra parte, en la comprensión de las necesidades y expectativas, no se pudo identificar información de terceros (empresas proveedoras, contratistas, subcontratas, colaboradores, visitantes, clientes, servicios médicos). En cuanto al establecimiento del alcance, dentro de sus 66 sedes o centros de enseñanza a nivel nacional no se incluyó el $27 \%$ de la certificación, es decir, 17 sedes no cuentan con certificación, que independientemente de que la institución principal y todas sus locaciones cumplen con la normativa legal en materia de seguridad y salud en el trabajo, sin embargo, no se muestra esa gestión que excede solo bastando cumplir con los requisitos legales. En ese sentido, INFOTESU puede tener la libertad y flexibilidad para establecer sus límites y aplicación al sistema de gestión de seguridad y salud en el trabajo, ya que esta norma es voluntaria y su credibilidad dependerá de la selección de sus límites, a fin de evitar impactos negativos o inducir a error a las partes interesadas. Respecto al sistema de gestión, va relacionado con la definición del alcance, es por ello, los resultados de cumplimiento, es decir, cuando abordas 
todos los procesos, integras los requisitos previstos, los programas, las competencias, puedes tener la confianza de controlar la gestión, y la acción o medida fue implementar para demostrar evidencia de cumplimiento.

En el liderazgo y participación de los trabajadores, se cuenta con un $85 \%$ de implementación, no se evidenció acciones sistemáticas orientadas a determinar cultura, es decir, valores, buenas prácticas de gestión, competencias, compromisos. Con referencia a la Política de la SST, no se aprecia la coherencia con otras políticas y estrategias de la institución; a fin de reflejar un sistema creíble y robusto. En cuanto a los roles, responsabilidades y autoridades en la institución, se realizó una encuesta evidenciando la falta de claridad en la comprensión de las responsabilidades, roles y autoridades para que lleguen a alcanzar los resultados de gestión preventiva y proactiva. Por ejemplo, la motivación y planes estratégicos para el reporte de condiciones de riesgo por los propios trabajadores. Concerniente a la propuesta y aportación de sugerencias e ideas de los trabajadores, si bien existen registros, sin embargo, no se hace seguimiento ni respuesta sobre los aportes "retroalimentación", contribuciones o ideas de los colaboradores; esta falta de control y gestión no será un factor importante para el éxito de su SGSST.

En la evaluación de la PLANIFICACIÓN se describen dos grandes subcapítulos, que son las acciones para abordar los riesgos y oportunidades y los objetivos para lograrlos, teniendo como resultado el $62 \%$. Dentro del sub capítulo de riesgos, no se pudo probar en tareas de riesgo alto en talleres eléctricos, talleres metal mecánica y talleres de automotriz el uso de registros de análisis de trabajo seguro (ATS); asimismo, la falta de atenuación del trabajo monótono en caso de docentes y personal administrativo, por otro lado, la falta de evaluaciones ergonómicas a fin de prevenir lesiones musculo esqueléticas. Este requisito es fundamental para que la seguridad total sea un proceso continuo, anticipándose a diversos escenarios cambiantes, evitando eventos, accidentes, enfermedades o daño a la reputación o imagen de la institución.

En cuanto a la identificación de peligros y evaluación de los riesgos y oportunidades, no se evidenció las actividades y situaciones no rutinarias que son ocasionales o no planificadas; como atender o responder accidentes, cuando la maquinaria se incendia, desastres naturales; asimismo, los peligros nuevos o modificados. Es imprescindible reconocer y comprender los peligros en las áreas de trabajo y por puesto de trabajo, a fin de eliminar o reducir los riesgos. En cuanto a los requisitos legales, no se evidenció el cumplimiento de requisitos legales de la ley de SST, sobre la Prevención de riesgos en su origen, para los casos de importación, cedan máquinas. Sustancia, útiles de trabajo, la falta de instrucciones, manuales y avisos de peligro o precaución en los esmeriles y taladros verticales (talleres). Otro rasgo de la planificación de acciones, no se ha incluido en su totalidad los sistemas de gestión de calidad, ambiental, anti soborno y financiero, para abordar cualquier cuestión que pueden impactar al SGSST.

Por otro lado, la falta de definición de objetivos específicos en el taller de metal mecánica sobre la reducción o eliminación de la exposición al ruido, la ausencia de objetivos proactivos y preventivos como la anticipación de riesgos. De esta manera, diseñar objetivos estratégicos, tácticos u operacionales del servicio de educación técnica. Del mismo modo, en la planificación debe contemplarse la asignación de recursos para priorizar la mejora de controles críticos.

En la evaluación del APOYO tuvo la implementación del $65 \%$, hallazgo que nos indica que existen muchos aspectos por mejorar, este gran capítulo exige 5 macros requisitos; empezando con los recursos identificándose condiciones de riesgo en los talleres, edificios y la falta de sistemas de contención de emergencias. En cuanto a la competencia, no se precisa claramente los requisitos de formación (complemento de la educación) de los docentes de talleres y personal de mantenimiento, esto asociado a los peligros y riesgos detectados. Habría que decir también, la falta de identificación competencias y conocimientos por cada trabajador(a), la práctica, las destrezas académicas y filológicas, la lectura, escritura, comunicación y la multiplicidad; como es el caso, en lugares donde se habla quechua $\mathrm{u}$ otros dialectos en los diferentes centros de formación del país. Además de la toma de conciencia, no se ha considerado en su totalidad la sensibilización, compromiso e involucramiento de los contratistas, proveedores, visitantes, a fin de generar una atmosfera de clima saludable y segura. Por otra parte, la comunicación se requiere actualizar la difusión de los principales contenidos, asegurando que los estándares de seguridad fluyan a todos los docentes, administradores, alumnos y partes interesadas. Hay que mencionar, además en la información documentada se debería controlar los documentos obsoletos y la identificación y control de documentos de origen externo, como catálogos de equipos de protección personal, sustancias químicas y de equipos y maquinarias.

En la OPERACIÓN, se cuenta con un bajo porcentaje de $58 \%$ a comparación con la muestra inicial que se tomó hace dos años (OHSAS 18001), toda vez que esta norma considera otros requisitos nuevos, sin embargo, no se evidenció controles de eliminación, sustitución o controles de ingeniería para los procesos de manipulación de productos químicos que usan para la enseñanza de talleres automotrices; asimismo, no se probó el análisis de la jerarquización de controles para demostrar si han evaluado los pasos desde la fuente, medio y receptor (reorganización del trabajo, protección de máquinas y caídas) y otras situaciones de exposición). Asimismo, la falta de establecimiento de mantenimientos preventivos o predictivos, los términos de referencia para las especificaciones de compra de bienes y servicios. De igual importancia, la falta de claridad en la gestión del cambio, pudiendo gestionar la definición de especificaciones de diseño de estaciones de estudio en los talleres, materias primas. En cuanto a la compra, la institución no verificó que los equipos y materiales adquiridos para el proceso de aprendizaje sean seguros para su manipulación. Para el caso de contratistas, cuentan con diversos proyectos de remodelación y construcción de edificios, ya que los centros de enseñanzas son de construcción antigua más de 40 años. Si bien, la gerencia de proyectos cuenta con contratos tercerizando los servicios de seguridad durante la obra, presentando un informe que señala que "transfiere 
los riesgos", es decir, tiene la mentalidad de que, al suceder un accidente grave o fatal, la institución se deslinda de toda responsabilidad. Esta última, es totalmente errado, toda vez que, por norma legal, la institución que contrate o sub contrate servicios, tiene responsabilidad garantizando los instrumentos, situaciones y circunstancia que protejan la seguridad y salud. Puesto que, ninguna empresa puede transferir los riesgos o responsabilidades o pensar que pueda eximir su responsabilidad legal. Se puede señalar, que la preparación y respuesta ante emergencias; no se ha considerado las indagaciones, hallazgos y circunstancia de mejora, en diversos informes de simulacros, donde se resaltaba obstrucciones de accesos de evacuación, las sirenas no operativas y puntos de reunión bloqueados por autos que utilizaban como parqueo.

En relación con la EVALUACIÓN DEL DESEMPEÑO, se cuenta con bajo porcentaje de 55\%, no obstante, no se pudo tener evidencia de los registros de quejas por temas de salud asistencial y ocupacional, caso dolencias y seguimiento de administrativos y docentes con enfermedades preexistentes, aproximadamente 14 colaboradores expusieron sus documentos médicos y correos donde no recibieron retroalimentación, esto conlleva a potenciales o probables eventos de salud o accidentes de trabajo. Además, se detectó equipos de monitoreo de ruido y temperatura descalibrados sin registro vigente de calibración ni verificación. En definitiva, este requisito es fundamental para alcanzar los resultados proyectados dentro del sistema de gestión en seguridad y salud ocupacional, para analizarlos, medirlos y tener controlado los procesos. Asimismo, para la evaluación del cumplimiento legal. Otro punto es, la auditoría interna se detectó que existen programas de los 4 últimos años donde se programa los mismos meses, por ejemplo, mayo y octubre. Esta forma de planear incumple con el espíritu de la norma, toda vez que se debe analizar tomando en cuenta los principales riesgos, criticidad de los procesos y los niveles de accidentabilidad, es decir, que si se puede programar en cualquier fecha y establecer mayores o menores frecuencias para verificar eficacia del sistema de gestión. En cuanto a la revisión por la dirección, se probó que no se contaba con conclusiones ni recomendaciones de algunos elementos de entrada tales como resultados de auditorías ni acciones correctivas por no conformidades recurrentes de controles operacionales de riesgo alto.

Por último, el capítulo de la norma es la MEJORA, que cuenta con el 57\% de implementación, no se evidenció acciones sistemáticas orientadas a la mejora, como es el caso de la falta de acciones de los resultados del análisis y evaluación del desempeño de la gestión de capacitación, talleres con seguridad, orden y limpieza. Con respecto a los sucesos llamado casi accidentes, accidentes de trabajo, incumplimientos o trasgresiones a los criterios normativos y acciones de mejora con análisis de causas. no se pudo evidenciar registros de reportes de actos o condiciones de riesgo, que esto ayudaría a ser una empresa proactiva, preventiva y de seguridad total. En cuanto a las no conformidades se detectó con mayor incidencia la falta de protección mecánica y eléctrica, falta de señalización y orden y limpieza. En cambio, en los incidentes no se detectó las pérdidas auditivas y presencia de humos metálicos en los docentes y alumnos que realizan aprendizaje y prácticas en los talleres de metal mecánica y soldadura. En relación a las acciones correctivas, tal como se señaló, no se evidencia un adecuado análisis de la jerarquía de controles, donde la institución priorice desde la eliminación, sustitución o controles de ingeniería en procesos críticos como son los talleres de metal mecánica, soldadura, automotriz y los trabajos de mantenimiento y obras civiles de terceros o contratistas.

En resumen, la calificación global de la gestión de seguridad y salud para una institución de enseñanza técnica superior fue del $60 \%$, que significa tener un nivel medio de cumplimiento, nos indica que aún hay más muchos requisitos por implantar, conservar, perfeccionar y renovar en favor de la administración de la seguridad y salud laboral en la institución. En la tabla 1, se observa un resumen del diagnóstico de la gestión de seguridad y salud en el trabajo realizada a la institución.

\section{2. Índice de siniestralidad o accidentabilidad \\ 3.2.1 Índice de frecuencia}

La tabla 2, muestra que el Índice de frecuencia de accidentes de alumnos, contratistas y trabajadores se elevaron en el 2017 pero disminuyeron en el 2018, además los alumnos presentaron los índices más bajo en los 3 años.

\subsection{2 Índice de gravedad}

El Índice de gravedad de accidentes de los alumnos y trabajadores tuvieron un ligero decrecimiento. Sin embargo, el decrecimiento de los contratistas fue notable, dado que ellos tuvieron el índice más alto en el 2017 pero éste se niveló al de los demás en el 2019.

\subsection{3 Índice de accidentabilidad}

La tabla 3, muestra que el Índice de accidentabilidad de alumnos y trabajadores tuvieron un ligero decrecimiento. Sin embargo, el decrecimiento de los contratistas fue notable, dado que ellos tuvieron el índice más alto en el 2017 pero este se niveló al de los demás en el 2019.

\subsection{Impactos}

\subsubsection{Propuesta para la solución del problema}

Se puede señalar, que los resultados del sistema de gestión de seguridad y salud ocupacional en INFOTESU, cuenta con un bajo porcentaje, debido a diversos requisitos que no se implementan y, por ende, probablemente no podrá ser un sistema de gestión maduro. Desde luego, modelar una cultura preventiva, mediante la composición de diversos elementos que interactúan entre sí, como son los espacios, tiempos, extensiones y alcances de gestión. Este modelo o propuesta de mejora tendrá un enfoque de la seguridad y salud en el trabajo de forma integral, con la finalidad de: a) Tratar de forma integrada los aspectos físicos, estado psicológico emocional y social de la seguridad y salud ocupacional; b) incorporar la seguridad en todos los alcances de gestión de la institución técnica superior, e c) Involucrar a todos los agentes de la institución educativa.

Además, el modelo de seguridad total o integral propuesto se estructura en cuatro componentes, basado 
Tabla 1. Resultados de la Gestión en Seguridad y Salud Ocupacional

\begin{tabular}{lcc}
\hline \multicolumn{1}{c}{ Numeral de la norma } & \% logrado de implantación & Actividades por ejecutar \\
\hline Entorno de la empresa & & Implementar \\
Visión de la empresa y su entorno & $54.50 \%$ & Mantener \\
Entender las exigencias necesidades e intereses de los colaboradores y terceros & \\
Establecimiento del campo de aplicación del sistema de gestión de la SSO & $85.00 \%$ \\
Estructura de la gestión de la SSO como sistema & \\
\hline Conducción e involucramiento de los trabajadores & \\
Conducción, convenio y acuerdo a los compromisos & \\
Directriz de la SST & \\
Funciones, tareas, labores, obligaciones y dirección de la empresa & \\
Sugerencias y colaboración de los trabajadores & \\
\hline Planeación & \\
Gestiones para Acciones para afrontar los riesgos y circunstancias & \\
Información básica & \\
Detección de peligros, valoración de riesgos y circunstancias & \\
Identificación y establecimiento de normas legales y otras exigencias & \\
Planeación de gestiones & \\
Propósito de la gestión de SST y planeación para obtener resultados & \\
Propósitos y metas de la SST \\
Planeación para obtener resultados de objetivos y metas de la SST
\end{tabular}

\section{Soporte}

Subvención o provisión de recursos

Capacidades

Concientización

Diffusion

Información general

Diffusion interna

$64.00 \%$

Mejorar

Diffusion externa

Gestión documentaria

Información general

Establecimiento y renovación

Monitoreo de la gestión documentaria

\section{Actuación}

Planeación y monitoreo operativo

Información general

Reducir o erradicar peligros y riesgos en SST

Mejora del cambio

Adquisiciones de bienes y servicios

Formulación e intervención ante eventos o emergencias

\section{Valoración del rendimiento}

Monitoreo, indicadores, monitoreo del rendimiento

Información general

Valoración del desempeño

Procesos de auditoria interna

Información general

Programación y ejecución de auditoría interna

Evaluación por la gerencia

\section{Mejoramiento}

Información general

Casos de accidentes, casi accidentes, Incidentes, incidentes peligrosos, desviaciones y acciones

Mejoramiento continuo

\begin{tabular}{lc}
\hline Total resultado implementación & $63 \%$ \\
\hline Calificación global en la Gestión de Seguridad y Salud Ocupacional & Medio \\
\hline
\end{tabular}


Tabla 2. Índice de frecuencia anual según Alumnos, Contratistas y Trabajadores

\begin{tabular}{cccc}
\hline \multirow{2}{*}{ AÑo } & \multicolumn{3}{c}{ Índice de frecuencia (I.F) } \\
\cline { 2 - 4 } & Alumnos & Contratistas & Trabajadores \\
\hline 2017 & 2.034 & 4.924 & 4.610 \\
2018 & 0.683 & 0.908 & 1.332 \\
2019 & 0.553 & 0.685 & 1.015 \\
\hline
\end{tabular}

Fuente: Estadísticas de la Institución de Enseñanza Técnica Superior 2017-2019 (Ministerio de Trabajo y Promoción del Empleo (MTPE), 2016)

Tabla 3. Índice de accidentabilidad Anual según Alumnos, Contratistas y Trabajadores

\begin{tabular}{cccc}
\hline \multirow{2}{*}{ AÑO } & \multicolumn{3}{c}{ Índice de accidentabilidad (I.A) } \\
\cline { 2 - 4 } & Alumnos & Contratistas & Trabajadores \\
\hline 2017 & 0.005 & 0.116 & 0.015 \\
2018 & 0.000 & 0.048 & 0.011 \\
2019 & 0.002 & 0.006 & 0.009 \\
\hline
\end{tabular}

Fuente: Estadísticas de la Institución de Enseñanza Técnica Superior 2017-2019 (Ministerio de Trabajo y Promoción del Empleo (MTPE), 2016)

en el modelo realizado por Alfredo Cutuli, que incluye, la seguridad proactiva (el antes), la seguridad operativa (el durante), la seguridad pasiva (el cuándo) y la seguridad reactiva (el después). Priorizando e inculcando la implementación, permanencia y mejora de una seguridad proactiva y preventiva en los aspectos operativos, tácticos y estratégicos.

Este modelo que se presenta es el resultado de la revisión de la literatura, y de contrastar la opinión de varios autores. La normativa, el nivel de cultura preventiva, la cultura y propósitos enunciados en la plataforma documentaria de gestión, y las gestiones que se promuevan, influirán en determinar y conservar la administración de la seguridad de la institución educativa superior técnico.

La propuesta del modelo de seguridad integral que responde a satisfacer la magnitud física, psicológica y socialmente inspirado la seguridad, se describe a continuación: a) magnitud física de la prevención de accidentes y enfermedades ocupacionales, se refiere a la seguridad centrada en criterios relacionados a los ambientes de trabajo, condiciones de locación, equipos, materiales, maquinarias y servicios, b) Magnitud psicológica de la prevención, se refiere a la seguridad centrada a la personas como respecto, cuidado y autocuidado, buscando asegurar garantizar la previsión y tranquilidad de los trabajadores y sociedad en los integrantes del instituto superior técnica, c) Dimensión social de la prevención, se refiere a la seguridad basada en determinar las relaciones basadas en la obediencia y convicción entre los integrantes del instituto superior técnico. En cuanto al aspecto de los agentes en el modelo de seguridad educativo integral, este debe tener una participación activa, como a continuación se señala: a) Administración y directivos, que se involucran directamente por su responsabilidad, b) Coordinadores / responsables de prevención, que se involucran por la ocupación de un cargo, c) Personal docente y no docente, que se involucran de manera indirecta por ser trabajadores, d) Alumnos y familia, que se involucran por ser usuarios de la institución educativa. Y respecto al ámbito de gestión de la institución educativa, estos deben atender la administración de la provisión y ejecución de diversos recursos tales como equipos seguros de enseñanza, salones y talleres seguros, a fin de prevenir y proteger a las personas y propiedad. Y respecto a la división del modelo en cuatro componentes, como la a) seguridad proactiva: se representa a la corrección anticipada preventivamente en los sitios identificados, estimados como potencial de ocurrir eventos como accidentes de trabajo o enfermedades ocupacionales, puesto que se plantea un plan de acción correctiva, preventiva y proactiva. b) la seguridad ejecutante se describe a que las personas ya sean trabajadores (docentes, administrativos), alumnos, visitantes y terceros (clientes, proveedores contratistas y sub contratistas), mediante la sensibilización y capacitación se forma el autocuidado, a fin de que sean las primeras personas seguras en sus áreas de trabajo o los terceros sepan de los peligros y riesgos. c) prevención pasiva, se destaca por tener personal competente para desarrollar ante accidentes, incidentes, eventos o emergencias, con el objetivo de reducir lo minimizar las lesiones o daños. Lo que favorece de manera oportuna la reactivación de los procesos de servicios de educación y administrativos. d). la seguridad reactiva se representa a que después de haber ocurrido accidentes de trabajo o cualquier otro evento inesperado, recién se toma acciones para analizar las causas verdaderas y determinar medidas de control, a fin de que no se vuelva a repetir. Sin embargo, esta última, no asegura, ni garantiza una gestión preventiva (es decir, actuamos después de que haya eventos, muertos o heridos); no obstante, se debe tener en cuenta que los eventos, accidentes de trabajo o enfermedades ocupacionales pueden suceder, por más que se aborde con buenas prácticas de gestión, considerando que, será mucho mejor o se reducirá eventos o pérdidas cuando demostramos una seguridad total mediante el modelo planteado.

\subsubsection{Costo de implementación de la propuesta}

Se estima que los costos de la implementación estarán cubiertos por el presupuesto de la institución de enseñanza técnica superior, tal como se muestra en la tabla 4. 


\subsection{Recursos del área de seguridad y salud en el trabajo}

\subsubsection{Humanos}

- Un Jefe de Seguridad y Salud Ocupacional

- Tres coordinadores regionales de SST

- 24 prevencionistas de riesgos (incluyen con experiencia bombero, ingenieros)

- Dos médicos ocupacionales

- Tres Higienistas Ocupacionales

- Dos Supervisores de Respuesta a Emergencias

- Una Asistenta Administrativa

- Tres auditores preventivos de SST
- Un comunicador y diseñador para potenciar los contenidos y afiches sensibilizadores de manera permanente.

\subsubsection{Beneficios que aporta la propuesta}

Las investigaciones en torno al tema de seguridad y salud laboral se entienden actualmente como un hecho fundamental para las organizaciones, ya sean instituciones públicas o privadas, asociaciones, organismos no gubernamentales, sindicatos, etc.

Preocupación que nace a partir de la valoración de los riesgos laborales que se presentan, dado que la siniestralidad laboral aún se da en muchas organizaciones de diversas actividades económicas.

Tabla 4. Presupuesto de la gestión de seguridad y salud ocupacional Adjuntamos el presupuesto del área SST para el año 2019 y 2020

\begin{tabular}{lc}
\hline Descripción 1 & Soles \\
\hline Equipamiento: equipos para respuesta de emergencia, extintores, gabinetes contra incendio, conos, señalética. & $52,300.00$ \\
Equipos de protección personal y colectiva & $22,129.00$ \\
Capacitación, entrenamiento, campañas y ferias & $18,632.00$ \\
Costos de certificación, mantenimiento y auditoría de certificación internacional en SST & $25,137.00$ \\
Asesoría para insertar la cultura de seguridad y otras prácticas & $18,145.00$ \\
Diseño, impresión y difusión de afiches y banners & $16,835.00$ \\
Mejora de infraestructura: pisos, rampas, semaforización. & $63,492.00$ \\
TOTAL & $216,670.00$
\end{tabular}

Tabla 5. Propuesta de plan de trabajo estratégico para implementar el modelo de Seguridad Total Estrategia para sensibilizar: año 2019

\begin{tabular}{|c|c|c|}
\hline & Descripción 2 & Alcance / Público dirigido \\
\hline 1. & $\begin{array}{l}\text { Estrategia en la definición de temas de sensibilización: Requisitos legales; Seguridad Basada en el } \\
\text { Comportamiento y Talleres Lúdicos. }\end{array}$ & Jefe de Seguridad / Gerencia de RRHH \\
\hline 2. & Sensibilización de los temas asignados (por grupos) & $\begin{array}{l}\text { Directores, Gerentes, Comités de SST, } \\
\text { Docentes, Administrativos, Alumnos }\end{array}$ \\
\hline 3. & Implementación de los requisitos legales & $\begin{array}{l}\text { Directores, Gerentes, Comités de SST, } \\
\text { Docentes, Administrativos, Alumnos }\end{array}$ \\
\hline 4. & Implementación de la brecha existente de la norma ISO 45001 versus OHSAS 18001 & Docentes, Administrativos, Alumnos \\
\hline 5. & $\begin{array}{l}\text { Cultura humana: mejora de competencias por puesto de trabajo, observaciones de conductas y } \\
\text { comportamientos, monitoreo de salud mental, ergonomía del trabajo. }\end{array}$ & Docentes, Administrativos, Alumnos \\
\hline 6. & Gestión documentaria física y digital: Libro digital de la cultura segura & Docentes, Administrativos, Alumnos \\
\hline 7. & $\begin{array}{l}\text { Control de equipos, maquinarias y actividades: persona competente, inspección de herramientas, } \\
\text { protección mecánica y eléctrica, autorización de trabajos de alto riesgo, equipos de protección personal } \\
\text { y colectiva. }\end{array}$ & Docentes, Administrativos, Alumnos \\
\hline 8. & $\begin{array}{l}\text { Gestión de materiales e insumos seguros: control de exposición de riesgos de materiales peligrosos o } \\
\text { sustancias químicas, energía eléctrica, agua, cajas y otros activos. }\end{array}$ & Docentes, Administrativos, Alumnos \\
\hline 9. & Establecimiento de políticas de seguridad en toda su magnitud. & Docentes, Administrativos, Alumnos \\
\hline 10. & Gestión de infraestructura segura: instalaciones, condiciones físicas (ruido, iluminación, temperatura). & Docentes, Administrativos, Alumnos \\
\hline 11. & Supervisión, Inspección y Auditoría del SGSST & Docentes, Administrativos, Alumnos \\
\hline 12. & Gestión por indicadores proactivos y preventivos & Docentes, Administrativos, Alumnos \\
\hline 13. & Círculos de Seguridad, Un espacio con la seguridad & Docentes, Administrativos, Alumnos \\
\hline 14. & Revisión de actos y condiciones acordes al estándar y mejoras de cada proceso/área. & Docentes, Administrativos, Alumnos \\
\hline 15. & Diseño, impresión y difusión de afiches y banners & Docentes, Administrativos, Alumnos \\
\hline 16. & Mejora de infraestructura: pisos, rampas, semaforización. & Docentes, Administrativos, Alumnos \\
\hline
\end{tabular}


Entonces, la forma de reducir o prevenir los índices de siniestralidad o accidentabilidad es adoptando una cultura de seguridad, y que se logrará si tenemos un modelo de seguridad integral, que apueste por el desarrollo y la promoción de actitudes y valores entre sus actores claves $\mathrm{y}$ fomente procedimientos y actuaciones seguras en la dirección de tener un clima de bienestar general y calidad de vida.

En ese sentido, la gestión de la seguridad en las organizaciones públicas o privadas es un valor que debe ser incorporado como cultura, en nuestro caso en una institución de enseñanza técnica superior, y su aplicación debe ser un aspecto fundamental y no postergado para beneficio de la comunidad educativa.

\section{DISCUSIÓN}

Se realizó el diagnóstico de la evaluación de la gestión de seguridad y salud en el trabajo basado en la norma ISO 45001 y medir el cumplimiento de cada componente como son: el CONTEXTO DE LA ORGANIZACIÓN, se obtuvo una implementación del 51.5\%; en relación con el LIDERAZGO Y PARTICIPACIÓN DE LOS TRABAJADORES, se obtuvo una implementación del 85\%;

Acerca de la PLANIFICACIÓN, se obtuvo una implementación del 62\%; En relación con el APOYO, se obtuvo una implementación del $65 \%$; De igual modo la OPERACIÓN, se obtuvo una implementación del 58\%; En adición a la EVALUACIÓN DEL DESEMPEÑO, se obtuvo una implementación del 55\%; finalmente, en cuanto a la MEJORA, se obtuvo una implementación del $57 \%$; sumando un total del $63 \%$ de implementación como nivel medio. Se amplió la muestra a todas las sedes a nivel nacional, a fin de poder tener un correcto resultado y poder priorizar la implementación de estándares de gestión, partiendo desde un plan estratégico de seguridad y salud en el trabajo, donde los directores y gerentes muestren un rol activo con sus trabajadores (docentes y administrativos), asimismo, con la comunidad del alumnado. Se priorizará la gestión de riesgos, la identificación de peligros, evaluación de riesgos y la implementación de controles, a fin de poder abordar los principales riesgos como monotonía, trabajos prolongados de pie (bipedestación), desmontaje y montaje de vehículos, talleres de soldadura en la reducción de material particulado, sonometría y dosimetría de ruido, maquinaria pesada, entre otros. Asimismo, la gestión de mantenimiento preventivo y predictivo de equipos y maquinarias para la enseñanza. Se programará las auditorías internas entre 5 a 7 veces al año por procesos, áreas, centro de estudios, y se fortalecerá las campañas y programas de cultura segura, desde la casa, calle y centro de trabajo y/o estudio. Esto contempla el cumplimiento de las normas de bioseguridad por la pandemia mundial y emergencia sanitaria, para salvaguardar y garantizar los recursos y mejores condiciones que salvaguarden la vida, la salud, armonía y bienestar de los docentes, contratistas, visitantes y alumnos. Porque la seguridad y salud también con esta coyuntura impredecible, volátil, de incertidumbre y que tiene para mucho tiempo, se considera también dentro de las recomendaciones como el teletrabajo para la parte administrativa y docentes.
Otros puntos por mejorar son la documentación y la comunicación de las funciones, responsabilidades, la rendición de cuentas y autoridad. Así, como el establecimiento, implementación y mantención de uno o varios procedimientos para la toma de conciencia de las personas. Asimismo, otra mejora es de sistematizar por un software los reportes de actos y condiciones de riesgos en los salones (aulas), talleres, instalaciones, a fin de responder inmediatamente una condición o no conformidad, de esta manera tomar acciones inmediatas y correctivas (haciendo análisis de causas), del mismo modo, reportes en línea de incidentes, incidentes peligrosos, accidentes y resultados de inspecciones, de esta forma, responder proactivamente y preventivamente la gestión de la institución, para dar protección y prevención a los alumnos que son aproximadamente noventa mil, instructores docentes, personas administrativo, visitantes, proveedores, contratistas y sub contratistas. Puesto que, por la complejidad de los procesos, su biografía (más de 80 centros de enseñanza en todo el país) y su propia realidad genera lentitud en procesar la información con correos, archivos en Excel y otros.

En resumen, la calificación global de la administración de la seguridad y salud para una institución de enseñanza técnica superior fue del $63 \%$, que significa tener un nivel medio de cumplimiento, nos indica que aún hay más muchos temas pendientes por implementar y mantener en favor de la gestión de la seguridad y salud laboral en la institución de enseñanza técnica superior.

Por otro lado, está la propuesta de mejora del sistema actual de gestión de seguridad y salud en el trabajo, que está basado en la revisión de la literatura, donde su composición no puede ser únicamente garantizada en base a la normativa, sino que tratará de incluirse en el grupo de requisitos de gestión, alienación estructural y el buen ejercicio seguro de las instituciones de enseñanza técnica superior.

El modelo de gestión de seguridad total tratará de aportar con elementos que guíen a la colectividad educativa y a los equipos de dirección (gerentes. directores regionales y jefes), con la finalidad de orientarse a una planificación integral, dictando y adoptando compromisos y priorizando los desembolsos en la infraestructura, documentación y competencias para una gestión segura. Propone un conjunto de dimensiones (física, emocional y social), agentes (alumnos, directivos, familia, administrativo, coordinadores, entidades del entorno) y ámbitos de gestión (provisión de recursos humanos competentes en prevención, clima o atmósfera laboral favorable en los talleres, salones de clase y áreas administrativa y comunes, las mejoras en habilidades blandas de comunicación, empatía y respeto, la estandarización de tiempos seguros, y ambientes con estándares de trabajo seguro). Además, la propuesta del modelo se divide en cuatro componentes: seguridad proactiva, la seguridad operativa, la seguridad pasiva y la seguridad reactiva.

Finalmente, una de las estrategias es sensibilizar a la alta dirección en hacerles conocer los requisitos legales como conocimiento imperativo, luego, una capacitación en Seguridad Basada en el Comportamiento, indicadores 
de gestión y Talleres Lúdicos, como habilidades blandas para mejorar la comunicación (saber escuchar, trabajo en equipo, iniciativa, liderazgo) y resultados. Lograr cambios las actitudes y valores de los trabajadores, a fin de aumentar la eficiencia y competitividad de los procesos seguros y por ende disminuir a cero la accidentabilidad y enfermedades ocupacionales.

Por otro lado, lograr disminuir el pensamiento y la acción "reactiva", convocando mediante indicadores la participación, involucramiento y compromiso de la alta dirección y a todo nivel. Por otra parte, se debe reducir o eliminar gradualmente la "dependencia", es decir, que se piense que el área de seguridad es solo es responsable de la seguridad y salud de los trabajadores; mentalidad que se debe erradicar. Como contrapartida, se debe interiorizar la gestión "independiente", dando mayor énfasis en el autocuidado y lograr hábitos e iniciativas de mejora de la prevención y protección. Ahora bien, hasta lograr que cada trabajador sea seguro, lógicamente con el soporte técnico del área de seguridad y salud en el trabajo; cabe destacar, que se debe inyectar el sentimiento de orgullo en contribuir con una cultura de seguridad en ser guardián de los demás.

Por lo consiguiente, implementando, manteniendo y mejorando permanentemente la norma ISO $45001 \mathrm{y}$ otras buenas prácticas citadas en la descripción y discusión, para fortalecer las competencias por puesto trabajo, la infraestructura, maquinarias, materiales, insumos, inspecciones y comunicaciones sensibilizadoras potentes

\section{CONCLUSIONES}

- Se determinó luego del diagnóstico, análisis y procesamiento de la información, las siguientes conclusiones: La evaluación de la gestión en seguridad y salud en el trabajo en la institución de enseñanza técnica superior basado en la norma ISO 45001, presentó un nivel medio de implementación, observándose que el componente del ciclo PHVA requiere implementar la brecha existente acorde a los requisitos de la norma en los procesos de la institución.

- Se identificó en forma general que el índice de accidentabilidad de la institución de enseñanza técnica superior tuvo un comportamiento estable en el periodo 2017-2019 en los grupos evaluados (alumnos, trabajadores), en el grupo contratistas, el comportamiento fue decreciente. Por su parte el índice de frecuencia calculado tuvo un comportamiento decreciente el último periodo 2017-2019 en los grupos evaluados (alumnos, contratistas, trabajadores), y finalmente el índice de gravedad obtenido tuvo un comportamiento estable en el periodo 2017-2019 en los grupos evaluados (contratistas, trabajadores), en el grupo alumnos, el comportamiento fue decreciente.

- Las características de la propuesta de mejora del sistema actual de gestión de seguridad y salud en el trabajo de la institución de enseñanza técnica superior está focalizada en dimensiones (física, emocional y social), agentes (alumnos, directivos, familia, administrativo, coordinadores, entidades del entorno) y ámbitos de gestión (recursos materiales, el clima de centro y aula, las relaciones, la organización y gestión de los recursos humanos, la organización y gestión de tiempos y espacios, y la prevención de riesgos). Además, dividida en cuatro componentes: seguridad proactiva, la seguridad operativa, la seguridad pasiva y la seguridad reactiva. Se recomienda realizar un plan piloto para la ejecución del nuevo modelo de gestión de seguridad y salud en el trabajo para la institución de enseñanza técnica superior, ya que nos permitirá realizar los ajustes necesarios en caso se presenten dificultades o inconvenientes durante su ejecución. Además, de verificar su viabilidad presupuestal.

- Se identificó condiciones de riesgo mediante una evaluación a nivel de muestra, mostrando la siguiente justificación en la institución: Incumplimientos de algunos requisitos legales y directivas en SST., Incremento de accidentes de trabajo, alumnos y contratistas, potencial multas o penalidades en caso de accidentes, Incremento de la prima de seguros por aumento de siniestrabilidad. Infraestructura no estándar acorde a requisitos de INDECI o ahora llamado ITSE (inspecciones técnicas de seguridad de edificaciones), Incumplimientos de SST de Contratistas, Terceros y Proveedores, Peligros y Riesgos críticos detectados, Deficiencia en servicios de alimentación por riesgos biológicos, Señalización y rotulación no estándar e incompleta, Falta de estandarización de orden y limpieza, Pérdida de imagen por eventos no deseados, los Prevencionistas no cubren al 100\% dedicados a la SSO.

- Es relevante centrar los esfuerzos por lograr una cultura de seguridad total en INFOTESU, empezando desde el autocuidado hacia un cuidado colectivo, pensando en los demás o tu entorno laboral, sin importar si es un trabajador de la institución, alumno, administrativo, proveedor, cliente, visitante. Implementar campañas permanentes de "yo se cuidarme"; "cuidemos todos"; "somos responsables de la seguridad". Asimismo, programas de cultura de seguridad total llamados: "un momento con la prevención" y "un espacio con la seguridad", a fin de cumplir y exceder el capítulo de Liderazgo de la ISO 45001.

- Se concluye también que la institución debe incluir factores relacionados con la comunicación, la competencia, la fatiga, los equipos, los procedimientos y otros controles, asimismo, nueva tecnología; sugerencias y recomendaciones de las partes interesadas; nuevos conocimientos y comprensión de cuestiones relacionadas con la seguridad y salud en el trabajo; cambios en las capacidades o la competencia de los trabajadores; 


\subsection{Recomendaciones}

- Implementar un liderazgo visible que resurja desde la alta dirección (dirección nacional), gerencias y directores zonales en provincias, es decir, la gerencia general conjuntamente con el especialista de seguridad, medico ocupacional $\mathrm{y}$ personal de mantenimiento e infraestructura, realicen visitas avisadas y no avisadas, a fin de identificar oportunidades de mejora, esto debe plasmado en un plan y programa permanente, a fin de que esté anotado el mismo, además no sea algo improvisado, aislado, eventual, ocasional, fortuito, suelto, es decir anecdótico; sino planeado y constante: orientado a la mejora continua como seguridad total.

- Abordar en tres criterios de documentación, infraestructura y competencia, a fin de identificar riesgos y establecer medidas preventivas de SST.

- Establecer dentro del modelo de cultura de seguridad total e integral: visión, valores y misión desde la alta dirección, promover un comportamiento seguro en todos sus niveles, crear un entorno para la mejora en el desempeño seguro, propiciar proyectos de innovación de procesos que minimicen los riesgos y aumente la productividad, promover una comunicación franca sobre los peligros, riesgos y acciones en conjunto con la participación de los trabajadores.

- Fortalecer las competencias a los integrantes del comité de seguridad y salud en el trabajo, mediante temas de capacitación tales como: análisis de riesgos IPERC identificación de peligros, evaluación de riesgos y controles; funciones y responsabilidades del comité; investigación de accidentes, incidentes, incidentes peligrosos y eventos; prevención y protección de enfermedades ocupacionales; liderazgo y cultura de seguridad; reglamento interno de seguridad y salud en el trabajo.

- Implementar cartillas y listas de verificación de actos, conductas y comportamientos seguros en las actividades de los instructores (docentes), administrativos y estudiantes en plena formación.

- Implementar la metodología SOL seguridad, orden y limpieza, a fin de tener lugares limpios, seguros y saludables, que no solo ayudará a la productividad, sino también a la calidad de trabajo con armonía y clima laboral aceptable.

- Mejorar las instalaciones de accesos peatonales con rampas para personas con discapacidad en caso de silla de ruedas, iluminación, semáforos, cruceros peatonales, señales viales, letreros motivacionales al personal instructivos, administrativos, alumnos, para evitar atropellos.

- Abordar mediante un plan y programa permanente la: vigilancia Epidemiología en Salud Ocupacional de los trabajadores, monitoreo de los agentes físicos: Monitoreo de Sustancias Químicas, Agentes Biológicos: Evaluación de Agentes microbiológicos, entre otros, que forman parte de una cultura de higiene industrial $\mathrm{u}$ ocupacional. Todo este punto de higiene se requiere saber si se encuentra dentro de los límites máximos permisibles en los almacenes de la institución, talleres de enseñanza como textil, soldadura, metal mecánico, joyería, electricidad, electrotecnia, salas de computo, entre otros.

- Finalmente, dependerá mucho del respeto en la aplicación de los estándares de gestión de seguridad y salud en el trabajo como cultura preventiva; es decir, abordar el cumplimiento, mantenimiento, mejora e innovación de las buenas prácticas de trabajo seguro; anticiparse a los peligros y riesgos potenciales, tomar acciones proactivas y preventivas desde la estrategia, planes, programas, objetivos, metas, capacitaciones, tareas específicas o mejora de actos o condiciones seguras, de lo contrario, se tendrá los mismos resultados o tomar acciones reactivas como normalmente se ejecutan.

- Finalmente, la institución educativa superior técnica, debe considerar en sus procesos, los requisitos de las normas de bioseguridad, que comprende la responsabilidad indirecta con las familias, comunidad, calle y centro de trabajo, puesto que, la prevención y protección frente al COVID-19 y otras cepas, forman parte dentro de la Ley de Seguridad y Salud en el Trabajo, como es también el teletrabajo, ergonomía, riesgos psicosociales, entre otros. Por ejemplo, el control de ergonomía, salud mental, sedestación y otros peligros y riesgos de los docentes y alumnos de clases en casa.

- Por tal motivo, este caso estudio también puede servir como provecho o beneficio para las universidades e inclusive colegios o institutos de educación, que debe abordar una cultura integral segura, incluyendo la nueva normalidad con el COVID-19.

\section{AGRADECIMIENTOS}

Ana Isabel Kido Takamatsu (mi esposa), por su coherencia en sus recomendaciones, motivación y apoyo constante en mejorar.

Jorge Jave Nakayo: por su soporte profesional técnico en el desarrollo de la tesis del presente artículo.

Carlos Cabrera Carranza: por su orientación y consejo profesional en el desarrollo de la tesis

\section{REFERENCIAS}

Hernández-Sampieri, R., Fernández-Collado, C. y BaptistaLucio, P. (2014). Metodología de la investigación. https:// periodicooficial.jalisco.gob.mx/sites/periodicooficial. jalisco.gob.mx/files/me 
Ministerio de Trabajo y Promoción del Empleo - MTPE), \& Ministerio de Salud - MINSA. (2010). Diagnóstico situacional en seguridad y salud en el trabajo. Proyecto "Iniciativa Andina EnSeguridad y Salud En El Trabajo," 59. http://bvsper.paho.org/videosdigitales/matedu/20120125 salud_trabajo_diag.pdf?ua $=1$

Ministerio de Trabajo y Promoción del Empleo (MTPE). (2016) Anuario Estadístico. http://www2.trabajo.gob.pe/archivos/ estadisticas/anuario/Anuario_2016_020717.pdf
Organización Internacional del Trabajo. (2018). Seguridad y salud en el trabajo en los Países Andinos. OIT Lima, Seguridad. https://www.ilo.org/lima/temas/seguridad-y-salud-en-eltrabajo/lang--es/index.htm

SENATI-Servicio Nacional de Adiestramiento para el Trabajo Industrial. (2012). Reglamento interno de seguridad y salud en el trabajo. Comité Nacional de Seguridad y Salud En El Trabajo (CNSST), 1-31. https://waldocc.files.wordpress. com/2016/03/reglamento_interno_seguridad_salud_en_ trabajo_2013.pdf 
